\title{
Tecnologías Web 2.0 en el proceso de formación universitaria: programa de capacitación para favorecer el conocimiento y habilidades de los docentes
}

\author{
Esthela M. San Andrés ${ }^{1}$, Milagro C. Rodríguez², Marcos F. Pazmiño ${ }^{1}$ y Kevin M. Mero ${ }^{1}$ \\ (1) Universidad Técnica de Manabí, Facultad de Ciencias Informáticas, Departamento de Tecnologías de la Información \\ y Comunicación. Avenida Urbina 130105. Portoviejo. Ecuador. (Correo-e: esthela.sanandres@utm.edu.ec; \\ marcos.pazmino@utm.edu.ec; kevin.mero@utm.edu.ec) \\ (2) Centro de Investigaciones de la Sede Macas. Universidad Católica de Cuenca. Avenida de las Américas, Cuenca, \\ Ecuador (Correo-e: mrodrigueza@ucacue.edu.ec)
}

Recibido Jun. 25, 2021; Aceptado Ago. 25, 2021; Versión final Oct. 24, 2021, Publicado Feb. 2022

\begin{abstract}
Resumen
El objetivo del presente estudio fue examinar la efectividad de una estrategia de capacitación ejecutada con los profesores de la Universidad Técnica de Manabí (UTM) en Ecuador sobre sus conocimientos y habilidades de tecnologías Web 2.0, así como su uso en el proceso formativo universitario. La muestra fue de 374 profesores de la UTM. Los resultados iniciales develaron que no todas las potencialidades del uso de estas tecnologías eran explotadas, generalmente por falta de conocimiento para su empleo. Por ello, se implementó una estrategia de capacitación diseñada y aplicada en cursos con contenidos blogs, microblogs, wikis, redes sociales, videos streaming, y Zoom entre otros. La aplicación de la prueba estadística de Wilcoxon, antes y después de la aplicación de la estrategia, evidencio que se favorecieron los conocimientos y las habilidades de los profesores y el uso de las herramientas que conforman la Web 2.0. Se concluye que la estrategia de capacitación benefició las habilidades Web 2.0 de los docentes.
\end{abstract}

Palabras clave: tecnologías; información; formación universitaria; herramientas tecnológicas; Web 2.0; capacitación docente

\section{Web 2.0 technologies in university training processes: training program to promote professor's knowledge and skills}

\begin{abstract}
The main objective of the present study was to examine the effectiveness of a training strategy conducted with professors at the Technical University of Manabí (TUM) in Ecuador to assess their knowledge, skills, and use of Web 2.0 technologies. The sample consisted of 374 TUM professors. Initial assessment revealed that the full potential of Web 2.0 technologies were not exploited, generally due to lack of knowledge about their use. For this reason, a training strategy was designed and applied in academic courses containing content related to blogs, microblogs, wikis, social media, video streaming, Zoom, and others. Application of the Wilcoxon statistical test allowed comparing data obtained in the survey before and after the application of the strategy. The results showed that the applied strategy successfully improved professors' knowledge, skills, and use of Web 2.0 tools. It is concluded that the training strategy applied improved professors' Web 2.0 capabilities.
\end{abstract}




\section{INTRODUCCIÓN}

En la actualidad los docentes se enfrentan a un gran desafío relacionado con la introducción de las tecnologías que facilitan el aprendizaje autónomo, el personalizado y el colaborativo en la educación superior. Lo anterior obliga a repensar en el uso de un enfoque pedagógico diferente basado en la práctica, la reflexión y la movilización del conocimiento asociado al uso de las Tecnologías de la Información y Comunicación (TIC). Un profesor del siglo XXI, que tiene como responsabilidad educar a alumnos que ya dominan algunas habilidades para el uso de estas herramientas tecnológicas, debe estar capacitado para el uso de las TIC.

La alfabetización tecnológica es una aliada para sacar provecho de las herramientas digitales en la docencia. Su beneficio se extiende a docentes y estudiantes, quienes deben buscar oportunidades de alfabetización digital y la puesta en práctica de esta alfabetización para combatir la tecnofobia y redescubrir los aportes de las TIC en el proceso de enseñanza aprendizaje (Echeverría, 2014). Lo importante es considerar la forma en que estas tecnologías propician el desarrollo de nuevas prácticas educativas, más eficaces y pertinentes, lo que permite fortalecer el protagonismo en el cambio educativo que tienen los docentes. Esta necesidad requiere asumir la complejidad de las TIC, reconociendo, multiplicando y potenciando aquellas experiencias de aprendizaje que estas favorecen o mejoran en relación a las prácticas tradicionales de enseñanza. La utilización de esas herramientas facilitará las actividades del pensamiento de orden superior, desarrollando el pensamiento crítico de los estudiantes y la construcción de sus conocimientos (Daniela et al., 2018).

Las herramientas que ofrece la web 2.0, conocidas también como aplicaciones web de segunda generación, son una parte importante de las TIC que contribuyen al desarrollo de las estrategias cognitivas, esenciales para el aprendizaje autónomo de los alumnos, entre estas se pueden encontrar blogs, wikis, podcasts y redes sociales en línea, que brindan facilidades para: compartir, interactuar y colaborar (Sendag et al., 2015). La Web 2.0 se definió por diferentes autores y desde diferentes perspectivas (Alexander, 2006; Grosseck, 2009; Greenhow y Chapman, 2020), pero las definiciones del término Web 2.0 son muy discutibles, sin embargo, no se excluyen porque la Web 2.0 se refiere al uso social de la Web que permite a las personas colaborar, involucrarse activamente en la creación de contenido, generar conocimiento y compartir información en línea, lo esencial es el cambio del paradigma de comunicación, pasando de una comunicación unidireccional a una comunicación bidireccional (Costa-Sánchez et al., 2017).

En el escenario, del siglo XXI donde se ha impuesto el uso de las redes sociales y la mayoría de los estudiantes utilizan la tecnología móvil y poseen un teléfono inteligente, las universidades se muestran cada vez más conscientes de los retos que supone dar una formación de calidad y apuestan por incorporar a sus entornos de aprendizaje, distintas herramientas tecnológicas de la Web 2.0, para uso y beneficio, tanto, de sus docentes como de su población estudiantil (Ozcinar et al., 2020). Referente al uso de la Web 2.0, las investigaciones realizadas por Fonseca et al., (2014) y Arancibia et al., (2014) encontraron que las herramientas de la Web 2.0 sumadas a las estrategias didácticas cuando son adaptadas a los tipos de contenidos de las asignaturas que se enseñan, son valiosas para el docente ya que utilizando las mismas el profesor incorpora diferentes actividades dinámicas que le facilitan a los estudiantes comprender los conocimientos a través de formas innovadoras. Son herramientas que permiten fomentar entre los estudiantes el trabajo colaborativo y el trabajo individual, permitiendo que los jóvenes adquieran las competencias necesarias en el siglo XXI como: el pensamiento crítico, la colaboración, las competencias digitales, aprender a trabajar en redes, así como el uso de las tecnologías de información y comunicación a lo largo del proceso de aprendizaje.

El estudio de Boza y Conde (2015) en relación a la actitud de los profesores ante la Web 2.0, señala que en general los docentes universitarios consideran que la web 2.0 es un instrumento que se requiere utilizar para una enseñanza de calidad, apuntando que los docentes más jóvenes están más predispuestos para utilizarla. Además, mencionan que las herramientas de la web 2.0 son vistas como atractivas y novedosas para los estudiantes y, que la Web 2.0 hace que el trabajo tanto, para profesores como para alumnos resulte más fácil. La investigación de Mendieta et al., (2017), encontró que los docentes afirman tener algún conocimiento al menos a nivel de conceptos en relación a algunas herramientas de la web 2.0; sin embargo, con relación a su nivel de uso afirman que muy poco las aplican en su labor docente, excepto los profesores de menor edad, que manifiestan tener un mayor conocimiento y utilización de estas tecnologías. Esta investigación revela como aspecto importante la necesidad que tienen los docentes de capacitación para desarrollar un conocimiento más profundo sobre el uso educativo de estas herramientas, así como las relacionadas con la búsqueda de información para utilizarlas adecuadamente en las actividades de enseñanza aprendizaje.

En la actualidad producto de la pandemia del Covid-19, los docentes se han visto ante el compromiso de integrar las TIC ajustando y replanteando sus métodos de enseñanza a modo de crear nuevos contextos de aprendizaje enriquecidos por estas herramientas, en sintonía con las experiencias de sus educandos, con la forma en que ellos interactúan en el mundo moderno utilizando las redes sociales, desarrollando un nuevo 
lenguaje que les permita dialogar con estos aprendices del nuevo milenio, así por ejemplo Martínez-Clares et al., (2020) en su estudio sobre el uso del blog como herramienta docente y comunicativa, reafirman que los blogs son herramientas que propician la interacción y el aprendizaje.

Por otra parte, Darginavičienè y Navickienè (2021) exploraron las actitudes de los docentes universitarios hacia el uso de las tecnologías de la información (TI) para la enseñanza de idiomas extranjeros, revelando que en las condiciones actuales la mayoría de ellos expresaron una actitud positiva hacia el uso de nuevas tecnologías para la enseñanza. Por otro lado, Hordatt-Gentles y Haynes-Brown (2021) valoraron la transición a la enseñanza en línea llevada a cabo por los docentes de América Latina y el Caribe durante la pandemia de Covid-19 encontrando que los profesores requieren capacitación y deben ser apoyados y empoderados con conocimientos y competencias en TIC que les permitan tomar sus propias decisiones y diseñar sus espacios de aprendizaje.

Concordando con lo anterior, se hace necesario el perfeccionamiento permanente en esta área, que permita el desarrollo de competencias digitales en los docentes pasando desde una fase inicial en el que desarrollan capacidades básicas de uso de las TIC a fases especializadas de integración de las TIC en su práctica profesional para atender las exigencias actuales (San Andrés et al., 2019). Lograr el perfil profesional del docente de la educación superior según la opinión de Alvarez-Flores (2021) implica desarrollar competencias digitales que le permitan ejecutar las actividades dentro de su desempeño laboral.

Para que los profesores logren las competencias digitales a un nivel de dominio avanzado necesitan capacitaciones actualizadas que los preparen para aprender a enseñar con estas herramientas, entre ellas, las de la web 2.0 con el fin de utilizarlas adecuadamente para ofrecer a sus alumnos oportunidades de construir y reconstruir su aprendizaje a través de la comunicación, la interacción, el acceso a recursos digitales, la colaboración y la expresión de sus construcciones. Aquellos docentes comprometidos con la enseñanza a los estudiantes del nuevo milenio tienen que ser capaces de guiarlos en su viaje educativo a través de nuevas herramientas tecnológicas. Ejercer un liderazgo educativo eficaz en el siglo XXI requiere de una suficiente cultura digital y presencia en la web de los docentes

Este estudio tuvo como objetivo examinar la efectividad de una estrategia de capacitación ejecutada con los profesores de la Universidad Técnica de Manabí, sobre sus conocimientos y habilidades respecto de las tecnologías de la Web 2.0, así como el uso en el proceso formativo universitario de estas herramientas. Se indagó sobre la siguiente pregunta de investigación: ¿Existe alguna diferencia significativa entre el uso de las herramientas de la Web 2.0 en el proceso de enseñanza aprendizaje antes de aplicar la estrategia de capacitación y después de aplicada con los docentes que participan en el estudio?

\section{METODOLOGÍA}

El estudio se realizó con una muestra de 374 profesores de una población de 963 para un 95\% de confiabilidad y $5 \%$ de margen de error. La investigación se realizó en tres etapas. En la primera se hizo un diagnóstico para conocer el estado de la problemática. Una segunda etapa para implementar una estrategia de capacitación en base a un programa elaborado y una tercera etapa para aplicar nuevamente el cuestionario utilizado inicialmente y valorar los cambios y examinar la efectividad de la capacitación realizada con los docentes.

\section{Participantes}

La selección fue mediante un muestreo aleatorio estratificado, dividiendo a la población en los distintos niveles en los que imparten clases los docentes del 1ro al 10mo ciclo en las carreras que oferta la universidad, obteniendo submuestras proporcionales en cada ciclo, los mismos dieron su consentimiento por escrito para formar parte de los sujetos de la investigación. De los 374 sujetos elegidos, el $100 \%$ respondió la encuesta. La distribución de los participantes fue 4,5\% (17) son docentes de 1er nivel, $7,2 \%$ (27) son profesores de 2 do nivel; $13,6 \%$ (51) de 3er nivel; 5,8\% (22) tanto de 4 to como de 5 to nivel; $8,5 \%$ (32) de 6 to nivel; 5,8\% (22) de $7 \mathrm{mo}$ nivel; $16,5 \%$ (62) de 8 vo nivel; $12,3 \%$ (46) de 9no nivel y $19,5 \%$ (73) de $10 \mathrm{mo}$ nivel.

\section{Instrumentos}

Los datos se recopilaron a través de la técnica de encuesta con su instrumento el cuestionario que fue diseñado por los autores de este estudio tomando en cuenta la literatura relevante en el tema. El cuestionario incluye datos de los participantes (edad, genero, nivel en el impartían clases y otros), ítems para conocer disponibilidad de computadoras y acceso a internet y su percepción acerca del nivel competencial para el uso de la computadora y el internet. En una pregunta se indaga sobre el nivel de conocimiento y habilidad que tenían los profesores sobre las tecnologías Web 2.0, se utilizó una escala de Likert de 5 opciones: Mucho (5); Bastante (4); Suficiente (3); Poco (2); Nada (1) que permitió obtener una puntuación para cada ítem. 
Además, se incorporaron preguntas para conocer la preferencia de uso de las tecnologías de la Web 2.0 en clase (blogs, microblogs, wikis, redes sociales, videos streaming, webquests, documentos en línea, otros). También, una pregunta para valorar el criterio de los docentes sobre la frecuencia de utilización de las herramientas de la Web 2.0 para favorecen el aprendizaje colaborativo de los estudiantes, atendiendo a las posibilidades de colaboración que caracteriza a estas tecnologías. En esta pregunta se utilizó una escala de Likert de 5 opciones: Siempre (5); Casi siempre (4); Algunas Veces (3); Rara vez (2); Nunca (1).

Para la validez de contenido que pretendió determinar si los ítems o preguntas propuestas reflejan el dominio del contenido (conocimientos, habilidades o destrezas) que se desea medir (Urrutia-Egaña et al., 2015) se utilizó el criterio de expertos a través del método Delphi por su alto grado de fiabilidad, flexibilidad, dinamismo y validez (de contenido y otras), garantizando el anonimato, la heterogeneidad y la interacción y retroalimentación de los expertos. Participaron un total de 25 expertos, el 55\% con grado de Ph.D y el 45\% eran Master en áreas de especialización en educación y en tecnologías de la información y la comunicación. Luego de dos rondas el $85 \%$ de los ítems alcanzaron la categoría de muy adecuado y el $15 \%$ restante de bastante adecuado, con estos resultados se perfeccionó el cuestionario y se obtuvo una segunda versión. Posteriormente, se aplicó una prueba piloto a 150 docentes para tomar los datos y determinar la consistencia interna, encontrando un coeficiente de confiabilidad Alfa Cronbach de 0,89 para el cuestionario general, mientras que los valores variaron entre un 0,80 a 0,90 en las preguntas ó items.

En la segunda etapa de la investigación, tomando en cuenta los resultados obtenidos en el diagnóstico inicial, se elaboró una estrategia de capacitación basada en un programa que fue sometido a criterio del Centro de Capacitación y Educación Continua de la Universidad Técnica de Manabí. El programa fue analizado por una comisión designada con docentes de la Facultad de Informática para valorar las herramientas de la Web 2.0 a incluir y por docentes de la Facultad de Ciencias de la Educación con la finalidad de analizar los aspectos pedagógicos de la integración de estas tecnologías en el proceso de enseñanza aprendizaje.

En la tercera etapa de la investigación se aplicó el mismo cuestionario utilizado inicialmente, para valorar los cambios en cuanto al nivel de conocimientos y habilidades sobre las herramientas de la Web 2.0 , de los docentes y sus criterios acerca del uso de estas tecnologías para favorecer el aprendizaje de los estudiantes. Los resultados obtenidos de las preguntas relativas al uso de las tecnologías de la Web 2.0 por parte de los profesores en el proceso de enseñanza aprendizaje se sometieron a un análisis en SPSS con la aplicación de la prueba estadística de los rangos con signos de Wilcoxon para datos apareados con las apreciaciones de su utilización en cada uno de los niveles o ciclos de las carreras donde impartían clases los encuestados; antes y después de aplicar el proceso de capacitación. Se utilizó un nivel de significación $\alpha=0,05$.

\section{RESULTADOS}

Los datos obtenidos, en la primera etapa referida al diagnóstico inicial indicaron que los participantes tenían entre 25 y más de 64 años con una media de 48 años. En relación al género de los participantes el 68,2\% (255) fueron hombres y el 32 \% (119) eran mujeres, debido a la distribución propia de la población. Los resultados de la indagación se organizan según las preguntas de investigación y se presentan a continuación los obtenidos antes de aplicar la estrategia de capacitación.

Todos los participantes tenían computadoras para trabajar con acceso a internet, ya fueran personales ó de uso en las instalaciones de la universidad, así como disponían de una cuenta de correo electrónico personal ó institucional. Además, referente al nivel de uso de la computadora e internet por los participantes, el $1 \%$ señaló que era poco competente, el 3\% consideró ser algo competente, el $46 \%$ mencionó ser competente y el $50 \%$ se consideró bastante competente. En la pregunta sobre el nivel de conocimiento y habilidad que tenían los profesores sobre las tecnologías Web 2.0, los resultados obtenidos antes de realizar la intervención con la capacitación fueron: Mucho (10\%), Bastante (20\%), Suficiente $(40 \%$ ) y el $30 \%$ de ellos eligió que conocían poco a estas tecnologías. En la pregunta relacionada con la frecuencia con que los profesores utilizaban las herramientas de la Web 2.0 para favorecer el aprendizaje colaborativo de los estudiantes en clases, los resultados sugirieron que antes de implementar la capacitación el 7,2\% de los profesores señaló que siempre utilizaba estas tecnologías para favorecer el aprendizaje colaborativo; el $11,8 \%$ dijo que casi siempre; el $31 \%$ manifestaron que algunas veces y más del $50 \%$ prácticamente no las utilizaba; el $37,7 \%$ dijo que rara vez y el $12,3 \%$ que nunca.

En la segunda etapa se elaboró la estrategia de capacitación basada en el diseño y ejecución del programa elaborado que constó de cinco cursos, el cual tuvo el siguiente objetivo general: desarrollar en los docentes de la Universidad Técnica de Manabí habilidades digitales, con énfasis en las herramientas de la web 2.0 como recurso tecnológico para la mediación pedagógica en la enseñanza presencial y virtual. Los resultados de aprendizaje definidos en el programa a alcanzar por los docentes que participaron en la capacitación fueron: conocer los fundamentos generales de la web 2.0; elaborar documentos compartidos que integren el uso de diferentes funciones básicas disponible en el procesador de texto online; utilizar los recursos de la web 
2.0 como herramienta de soporte en el desarrollo de sus actividades académicas y de investigación; realizar presentaciones utilizando las diferentes herramientas que posee la plataforma web como sustento básico en sus trabajos expositivos dentro del programa y para sus clases; crear videos animados de la clase como apoyo audiovisual para el docente en sus asignaturas, las aplicaciones de videoconferencias entre otros.

En la estrategia planteada se consideró la ejecución del programa para lo cual se elaboró un cronograma que abarcó el segundo semestre de 2019 y el primer semestre del 2020. Los profesores que actuaron como facilitadores de las distintas unidades de los curso todos contaban con títulos de 4to nivel Magister ó Ph.D, en ciencias informáticas ó ciencias de la educación y la preparación suficiente en estos temas. La realización de los cursos en el primer período (Julio-Diciembre 2019) fue en modalidad presencial con duración de tres meses cada curso y una frecuencia semanal de 2 horas cada una, aquí se abordaron en el curso uno: los blogs, los microblogs, y las wikis; en el curso dos: las redes sociales, los videos streaming y las WebQuests. En el segundo período en los meses de enero, febrero se ejecutó de forma presencial el curso tres sobre el trabajo con documentos en línea en Google Docs, para potenciar el trabajo coolaborativo.

A partir de marzo debido al cierre de las universidades producto de la pandemia se continuó trabajando de forma virtual a través de videoconferencias en línea de manera sincrónica dos veces a la semana con 2 horas de duración, continuando con el cuarto curso que fue dedicado a las aplicaciones para videoconferencias como BigBlueButton, Zoom y otras, debido a la necesidad que enfrentaban los profesores para hacer frente a los retos de la pandemia del Covid-19, y mantenerse ofreciendo la enseñanza a sus estudiantes. En el quinto curso se abordaron las pizarras colaborativas digitales; herramientas para elaborar mapas mentales colaborativos como MindMeister y las herramientas para la comunicación visual como Powtoon y Prezi. Todos los cursos estuvieron acompañados de la parte virtual en la plataforma Moodle donde los participantes intercambiaban de forma asíncrona y entregaban sus tareas fundamentalmente dirigidas a la aplicación de las herramientas estudiadas en las clases de las asignaturas que enseñaban. Se acreditó a los docentes 120 horas de capacitación por su participación en el programa, válidas en su currículo para el escalafón.

En la tercera etapa de la investigación, después de realizada la capacitación los resultados en la aplicación del cuestionario arrojaron los siguientes datos: La mayoría de los participantes consideró que después de la capacitación su nivel de conocimientos y habilidades relacionados con las herramientas de la Web 2.0 aumentaron obteniéndose Mucho (40\%); Bastante (26\%); Suficiente (15\%) y sólo el $19 \%$ de ellos pensó que seguían conociendo poco a estas tecnologías. Los resultados obtenidos de las preguntas relativas al uso de las tecnologías de la Web 2.0 por parte de los profesores en el proceso de enseñanza aprendizaje se sometieron a un análisis con la aplicación de la prueba estadística de rangos con signos de Wilcoxon para datos apareados con las apreciaciones de su utilización en cada uno de los niveles o ciclos de las carreras donde impartían clases los docentes encuestados antes y después de aplicar el proceso de capacitación. Se utilizó un nivel de significación $\alpha=0,05$. Este proceso reveló lo siguiente tomando en cuenta cada una de las herramientas de la Web 2.0 que fueron sometidas al criterio de los profesores para valorar su uso en el proceso de enseñanza aprendizaje.

En el caso del Blogs, el valor del estadístico para los datos de la muestra calculado a través del SPSS fue de Sig. asintótica bilateral $=0,005$ y el valor de significación asumido $\alpha=0,05$, considerando la hipótesis nula $\mathrm{H}_{0}$ como la aseveración de que No hay una diferencia entre los resultados de la utilización en el proceso de enseñanza aprendizaje de la Herramienta blogs de la WEB 2.0 antes y después de aplicar el Programa de Capacitación y la hipótesis alternativa $\mathrm{H}_{\mathrm{a}}$ como la aseveración de que Hay una diferencia entre los resultados de la utilización en el proceso de enseñanza aprendizaje de la Herramienta blogs de la WEB 2.0 antes y después de aplicar el Programa de Capacitación, al ser Sig. asintótica bilateral $=0,005<0,05=\alpha$, se rechaza la hipótesis nula y se plantea que existe suficiente evidencia estadística para sustentar la aseveración de que hay una diferencia entre los resultados respectivos al uso del blogs, antes y después de recibir la capacitación con el programa ideado por el proyecto de investigación (Triola, 2009).

Respecto al uso en el proceso de enseñanza aprendizaje de las herramientas: Wikis, Redes Sociales, Videos streaming, Documentos en Línea, Google Drive, Kahoot, Mindmeister, Padlet, PowToon, Prezi, Zoom, según se muestra en la tabla 1, el valor correspondiente en cada caso al valor de Sig. asintótica bilateral, para los datos de la muestra es menor que el nivel de significación asumido $\alpha=0,05$, por lo que se rechaza la hipótesis nula $\mathrm{H}_{0}$ y se ha aceptado Ha: como la aseveración de que hay una diferencia entre los resultados de la utilización en el proceso de enseñanza aprendizaje de cada una de estas Herramienta de la WEB 2.0 antes y después de aplicar el Programa de Capacitación y se plantea que existe suficiente evidencia estadística para sustentar la aseveración de que hay una diferencia entre los resultados respectivos al uso de cada herramienta, antes y después de recibir la capacitación con el programa ideado y aplicado como parte de las actividades del proyecto de investigación ejecutado por el equipo de investigadores, para responder a las exigencias actuales de la educación superior. 
Tabla 1: Resultados de la Prueba de rangos con signos de Wilcoxon para los datos de la muestra, respecto al uso de las distintas herramientas de la Web 2.0

\begin{tabular}{|c|c|c|}
\hline Herramientas de la Web 2.0 & $\begin{array}{c}\text { Valor Sig asintótica } \\
\text { para datos de la muestra }\end{array}$ & Nivel de significación \\
\hline Wikis & $=0,008$ & \multirow{3}{*}{$\alpha=0,05$} \\
\hline Redes Sociales & $=0,005$ & \\
\hline Videos streaming & $=0,007$ & \\
\hline Documentos en Línea & $=0,005$ & \multirow{8}{*}{$\begin{array}{l}\text { Este valor de } \alpha \text { es el mismo para todas las herramientas } \\
\text { ya que se ha asumido el mismo nivel de significación en } \\
\text { todos los casos }\end{array}$} \\
\hline Google Drive & $=0,005$ & \\
\hline Kahoot & $=0,008$ & \\
\hline Mindmeister & $=0,005$ & \\
\hline Padlet & $=0,005$ & \\
\hline PowToon & $=0,005$ & \\
\hline Prezi & $=0,014$ & \\
\hline Zoom & $=0,005$ & \\
\hline
\end{tabular}

A pesar de los resultados anteriores los datos obtenidos en la aplicación de la prueba de Wilcoxon a la utilización de otras tecnologías de la Web 2.0, como: microblogs, WebQuests, BigBlueButton, Google Site, podcasts, en la tabla 2 se muestra que estas han sido menos utilizadas y que el programa de capacitación no ha provocado una diferencia significativa desde el punto de vista estadístico en cuanto a su implementación en el proceso de enseñanza aprendizaje por parte de los profesores que formaron parte de la muestra.

Tabla 2: Resultados de la Prueba de rangos con signos de Wilcoxon para los datos de la muestra, respecto al uso de otras herramientas de la Web 2.0

\begin{tabular}{|c|c|c|}
\hline Herramientas de la Web 2.0 & $\begin{array}{c}\text { Valor Sig asintótica } \\
\text { para datos de la muestra }\end{array}$ & Nivel de significación \\
\hline MicroBlogs & $=0,053$ & \multirow{3}{*}{$\alpha=0,05$} \\
\hline WebQuests & $=0,052$ & \\
\hline BigBlueButton & $=0,059$ & \\
\hline Google Site & $=0,056$ & \multirow{2}{*}{$\begin{array}{l}\text { Este valor de } \alpha \text { es el mismo para todas las herramientas } \\
\text { ya que se ha asumido el mismo nivel de significación en } \\
\text { todos los casos }\end{array}$} \\
\hline Podcasts & $=0,066$ & \\
\hline
\end{tabular}

Se observa en la tabla 2, que los valores de Sig. asintótica bilateral calculados para los datos de la muestra en cuanto al uso de cada una de las herramientas de la Web 2.0 que se señalan, son mayores a $\alpha=0,05$ por lo que se acepta $\mathrm{H}_{0}$ como: "No hay diferencias entre los resultados de la utilización en el proceso de enseñanza aprendizaje de las Herramienta MicroBlogs; WebQuests; BigBlueButton; Google Site; Podcasts de la WEB 2.0 antes y después de aplicar el programa de capacitación con los docentes". En este sentido es importante mencionar que la capacitación no abarcó una profundización en cada una de las herramientas mencionadas en las tablas 1 y 2, por lo que la aplicación de la prueba estadística evidencia donde los profesores requieren una mayor preparación respecto al uso de algunas de estas herramientas en el proceso de enseñanza aprendizaje y alertó a los investigadores sobre las mejoras o perfeccionamiento que debe tener la proyección de la capacitación cuando se vaya a extender a todos los profesores de la universidad.

Otros resultados revelaron que la puntuación más alta en el nivel de uso de tecnologías Web 2.0 fue Redes sociales (23,6\% antes), (48,1\% después de la capacitación); Videos streaming (10,5\% antes), (20,8\% después de la capacitación); Documentos en línea ( $24,6 \%$ antes), (50,4\% después de la capacitación). Respecto al uso de las herramientas de la Web 2.0 para favorecer el aprendizaje colaborativo de los alumnos, los resultados después de la aplicación del programa de capacitación se observan en la figura 1. Se muestra que el $43,3 \%$ señaló que siempre las utilizaba; el 40,9\% mencionó que casi siempre; el 11,2\% dijo que algunas veces hizo uso de estas herramientas y solo un $4,5 \%$ dijo que rara vez las utilizaba no presentándose ningún caso que señalara que nunca hacia uso de estas tecnologías. 


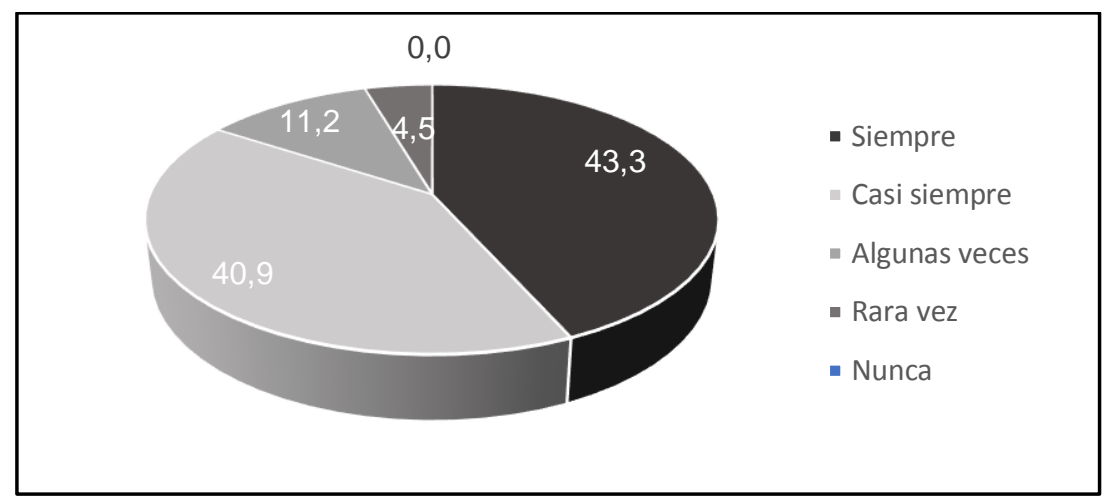

Fig. 1: Frecuencia de uso de las herramientas de la Web 2.0 para favorecer el aprendizaje colaborativo

\section{DISCUSIÓN}

La información derivada del análisis de los datos aportados por la investigación mostró que los profesores mejoraron sus conocimientos y habilidades relacionados con las herramientas de la Web 2.0 donde la mayoría de los docentes los consideraron entre mucho y suficiente (81\%) después del programa de capacitación, corroborando lo planteado por (Mendieta et al., 2017) y (Caliskan et al., 2019) sobre la necesidad que tienen los docentes de capacitación para desarrollar un conocimiento más profundo sobre estas herramientas. El nivel en el uso de tecnologías Web 2.0 después de la capacitación, según el criterio de los docentes fue alto, especialmente con ciertas tecnologías, como las redes sociales, Videos streaming, Documentos en línea, para favorecer el aprendizaje colaborativo en clase lo que concuerda con los hallazgos de la investigación de Zakaria et al., (2012) y Sari (2019) quienes reporta que el uso de las redes sociales y otras herramientas de la Web 2.0 ofrecen beneficios al proceso de aprendizaje permitiendo la colaboración entre las personas, el desarrollo de destrezas de comunicación y de investigación, además de un incremento de la motivación.

Si bien el uso de otras como: microBlogs, WebQuests, BigBlueButton, Google Site, podcasts son limitados, la mayoría de los profesores reconocen el valor de emplear tecnologías Web 2.0 en la instrucción. Los docentes consideraron que el tiempo y la capacitación son factores importantes. Además, opinaron que con estas herramientas se beneficia la interacción profesor-estudiante criterios que son coincidentes con los de Rogers (2014). Los resultados de este trabajo apoyan los hallazgos de investigaciones previas sobre este tema como los de Echeng y Usoro, (2014) y Ozcinar et al., (2020) cuyos resultados demuestran que las herramientas de la Web 2.0 pueden mejorar la participación, colaboración e interacción en el aprendizaje. Por otra parte, los hallazgos de esta investigación corroboran los obtenidos en otros estudios donde se plantea que conocer el nivel de las competencias digitales de los profesores propicia una comprensión de las necesidades de aprendizaje profesional que requieren para facilitar la incorporación de estrategias tecnopedagógicas que impulsen la innovación en la educación del siglo XXI. (Basantes-Andrade et al., 2020)

\section{CONCLUSIONES}

De acuerdo a los resultados obtenidos, su análisis y discusión se plantean las siguientes conclusiones principales:

(1) la estrategia de capacitación tuvo un efecto positivo en los profesores mejorando sus conocimientos y el uso de las tecnologías de la Web 2.0. La mayoría de los profesores que participaron en la capacitación estarían dispuestos a utilizar tecnologías Web 2.0, por tanto, se puede concluir que los profesores con conocimiento y capacitación pueden utilizar las tecnologías Web 2.0 para apoyar los cursos en las asignaturas de los programas universitarios, ya que consideran que tienen potencial para mejorar el aprendizaje y asegurar la interacción entre los alumnos y profesores;

(2) Las tecnologías Web 2.0 son poderosos medios habilitadores y potencialmente útiles actualmente cuando están surgiendo nuevas exigencias a la educación superior. El estudio indica una voluntad por parte de los profesores de capacitarse para hacer uso de la Web 2.0; y

(3) La investigación brinda orientación sobre las características del diseño de la instrucción a los profesores que no son nativos digitales para que estos puedan apropiarse del conocimiento sobre estas herramientas y poder hacer uso en el proceso de enseñanza aprendizaje e insiste en la necesidad de continuar realizando investigaciones en este campo para perfeccionar los procesos de capacitación de los docentes en el conocimiento y uso de tecnologías en el proceso formativo universitario. 


\section{REFERENCIAS}

Alvarez-Flores, E. P., Uso crítico y seguro de tecnologías digitales de profesores universitarios, https://doi.org/10.4067/S0718-50062021000100033, Formacion Universitaria, 14(1), 33-44 (2021)

Alexander, B., Web 2.0: A New Wave of Innovation for Teaching and Learning?, Educause, Marzo-Abril, https://www.educause.edu/ir/library/pdf/erm0621.pdf (2006)

Arancibia, M., Cárcamo, L., y otros 3 autores, Re-pensando el uso de las TIC en educación: Reflexiones didácticas del uso de la Web 2.0 en el aula escolar, https://doi.org/10.3989/arbor.2014.766n2014, Arbor, 190(766), 1-13 (2014)

Basantes-Andrade, A. V., Cabezas-González, M., y Casillas-Martín, S., Competencias digitales en la formación de tutores virtuales en la Universidad Técnica del Norte, Ibarra-Ecuador, https://doi.org/10.4067/S071850062020000500269, Formacion Universitaria, 13(5), 269-282 (2020)

Boza, Á., y Conde, S., Web 2.0 en educación superior: formación, actitud, uso, impacto, dificultades y herramientas, Digital Education Review, ISSN 2013-9144, 28, 45-58 (2015)

Caliskan, S., Guney, Z., y otros 3 autores, Teachers' views on the availability of web 2.0 tools in education, https:// doi.org/10.3991/ijet.v14i22.11752, International Journal of Emerging Technologies in Learning, 14(22), 70-81 (2019)

Costa-Sánchez, C., Rodríguez-Vázquez, A. I., y Direito-Rebollal, S., Docencia universitaria 2.0. Efectos de la Web 2.0 en la docencia universitaria como objeto de estudio de las revistas españolas de comunicación, https://doi.org/10.4185/RLCS-2017-1220, Revista Latina de Comunicacion Social, 72, 1300-1316 (2017)

Daniela, L., Visvizi, A., y otros 2 autores, Sustainable higher education and technology-enhanced learning (TEL), https://doi.org/10.3390/su10113883, Sustainability (Switzerland), 10(3883), 1-22 (2018)

Darginavičienè, I., y Navickienè, V., Use of native language in learning english for specific purposes, https://doi.org/10.15181/tbb.v71i2.1104, Tiltai, 71(2), 109-124 (2021)

Echeng, R., y Usoro, A., Acceptance factors and current level of use of web 2.0 technologies for learning in higher education: a case study of two countries, https://doi.org/10.14569/IJACSA.2014.050502, International Journal of Advanced Computer Science and Applications, 5(5), 9-14 (2014)

Echeverría, A. C., Usos de las TIC en la docencia universitaria: opinión del profesorado de educación especial, Revista Electrónica Actualidades Investigativas En Educación, ISSN 1409-4703, 14(3), 1-24 (2014)

Fonseca, L., Medellín, L., y Vásquez, J., El uso de herramientas de la web 2.0 como estrategias didácticas en el proceso de enseñanza-aprendizaje de jóvenes universitarios, PAAKAT: Revista de Tecnología y Sociedad, ISSN 2007-3607, 4(7), 7 (2014)

Grosseck, G.,To use or not to use web 2.0 in higher education?, https://doi.org/10.1016/j.sbspro.2009.01.087, Procedia Social and Behavioral Sciences, 1, 478-482 (2009)

Hordatt-Gentles, C., y Haynes Brown, T., Latin American and Caribbean Teachers' Transition to Online Teaching During the COVID-19 Pandemic: Challenges, Changes and Lessons Learned, https://doi.org/10.12795/pixelbit.88054, Media \& Education Journal, 61, 131-163 (2021)

Martínez-Clares, P., Martínez-Juárez, M., y Pérez-Cusó, F. J., Los Blogs como recurso de la orientación profesional en la Web 2.0, Revista Española de Orientación y Psicopedagogía, ISSN 1989-7448, 31(3), 7-25 (2020)

Mendieta, C. M., Vázquez, E., y Cobos, D., Valoración de las competencias tecnológicas del profesorado universitario: Un estudio en la facultad regional multidisciplinaria de carazo, https://doi.org/10.21556/edutec.2017.60.843, Edutec Revista Electrónica de Tecnología Educativa, (60), 1-12 (2017)

Greenhow, C., y Chapman, A., Social distancing meet social media: digital tools for connecting students, teachers, in an emergency, https://doi.org/10.1108/ILS-04-2020-0134, Information and Learning Science, 121(5), 331-342 (2020)

Ozcinar, Z., Sakhieva, R.G., y otros 4 autores, Student's Perception of Web 2.0 Tools and Educational Application, https: //doi.org/10.3991/ijet.v15i23.19065, International Journal of Emerging Technologies in Learning, 15(23), 220-233 (2020)

Rogers, M., Web 2.0 Use in higher education, https://doi.org/10.2478/eurodl-2014-0024, European Journal of Open, Distance and E-Learning, 17(2), 130-142 (2014)

San Andrés, E., Pazmiño, M., Mero, K., y Pinargote, C., Las Herramientas de la web 2.0 en la mediación pedagógica universitaria, Universidad, Ciencia y Tecnología, ISSN 2542-3401, Especial(3), 111-121 (2019)

Sari, M. I.,The use of Web 2.0 tools for learning in EFL context: pre-service teachers' voice, https://doi.org/doi: 18196/ftl.4243, Journal of Foreign Language Teaching \& Learning, 4(2), 136-149 (2019)

Sendag, S., Ero, O., y otros 2 autores, Preservice teachers ' critical thinking disposition and Web 2 . 0 Competencies, https://doi.org/ 10.30935/cedtech/6148, Contemporary Educational Technology, 6(3), 172-187 (2015)

Triola, M. F., Estadística, 10ª 1-904, Pearson Educatión, ISBN 978-970-26-1287-2, México (2009)

Zakaria, M. H., Watson, J. A., y Edwards, S. L., The Adoption of e-learning 2.0 in higher education by teachers and students: An investigation using mixed methods approach, https://doi.org/10.7763/IJEEEE.2012.V2.90, International Journal of E-Education, e-Business, e-Management and e-Learning, 2(2), 108-112 (2012) 